\title{
Interfacial kinetics effects on transdermal drug delivery: a computer modeling
}

\author{
Malcolm M. Q. Xing ${ }^{1}$, Ning Pan $^{1}$, Wen Zhong ${ }^{2}$, Xiaoyin Hui ${ }^{3}$ and Howard I. Maibach \\ ${ }^{1}$ Department of Biological Systems Engineering, University of California, Davis, CA, USA, ${ }^{2}$ Departments of Medical Microbiology and Textile Sciences, \\ University of Manitoba, Winnipeg, MB, Canada and ${ }^{3}$ Department of Dermatology, University of California, San Francisco, CA, USA
}

\begin{abstract}
Background/purpose: Percutaneous permeation is a frequently used approach in drug delivery, but the detailed physics process in the patch - stratum corneum (SC) viable epidermis system remains unclear: the influence of the interphases in the multilayered structure has been little studied.

Methods: This paper applied the finite-element method to develop a contact algorithm with an interphase element to account for the interphase barrier on drug diffusion and chemical absorption during a transdermal drug delivery process. A more realistic multilayer structure, including the patch, SC and viable epidermis, are incorporated into the algorithm. Both interphases between the patch and SC, and between SC and viable epidermis are considered.

Results: Our study confirms that the interphase transfer coefficients have a direct connection with drug concentration and flux distribution along the diffusion paths. The
\end{abstract}

D RUG DELIVERY by percutaneous permeation is a common approach for administration of chemical therapeutic agents, which may not function well in the gastrointestinal tract or liver due to presystemic metabolism $(1,2)$. Chemical absorption into and permeation through the skin plays a key role in both dermatopharmacology and dermatotoxicology (3). Formulation of the medication, stratum corneum (SC), viable epidermis and dermis (containing circulation post-capillary) constitutes the primary dermatol - transport system.

The SC, the outermost skin layer, is a lipidprotein biphasic membrane structure punctured by protein-filled cronecytes whose chemical nature and geometrical tortuosity contribute to certain levels of diffusional resistance against penetration of substances including outer environmental toxicity and transdermal drugs (4).

Given the skin's structural and biochemical complexity, and the restrictions of in vivo and even in vitro experiments, mathematical analysis and modeling provide an attractive alternative, which, in spite of assumptions often grossly simulation results suggested a potential for the optimal control of drug diffusion. The partition coefficients and other interphase barrier factors can be incorporated into the model.

Conclusions: The algorithm can deal with complicated geometrical conditions, which is difficult using classical analytical approaches. Furthermore, calibrated against experiments, the model may predict more realistically the drug delivery process and drug distribution profiles so as to assist in the patch and even drug design.

Key words: transdermal drug - interphase barrier - multilayer finite-element model - contact transfer algorithm drug diffusion profiles

(c) Blackwell Munksgaard, 2007

Accepted for publication 26 May 2007 simplifying, may still offer some insights on the trend and influences of related factors. They have been proved to be valuable.

More specifically, a mathematical model for transdermal diffusion and permeation processes may provide the physics insight on the kinetics characteristics in diffusion and permeation, and the influences of such parameters as the types of chemical agents and the skin properties in both spatial and temporal regimes. So far, most existing models for drug transdermal release employ Fick's first and second laws to explain the release process in complex biological membranes like skin. According to Fick's first law, diffusive flux equals the negative product of the diffusion coefficient and the concentration gradient; here it is

$$
J=-D_{i j} \frac{\mathrm{d} C_{i}}{\mathrm{~d} x}
$$

where $D_{i j}$ is the binary diffusion coefficient of the solute $i$ in the solvent $j, C_{i}$ is the concentration of solute $i$ and $x$ is the gradient direction (5). 
Kalia and Guy (1) reviewed the mathematical models describing the drug release based on formulation scenarios. Roberts and Anissimov (6) summarized the percutaneous absorption models allowing for various boundary conditions associated with the solute transport across a membrane structure, including clearance from the receptor solution, and from the membrane and diffusion in an underlying layer. Hostynek et al. (7) provided a correlation of in vivo and in vitro percutaneous absorption with a mathematical model. Kubota and Maibach (8) proposed a compartment model, different from the diffusion model in finite-dose percutaneous permeation pharmacokinetics, to predict the lag times and steady-state flux. The intercompartmental transfer rate constants were also defined. Kubota et al. (9) employed a simple single-layer model to specify the permeability coefficient and the drug amount in skin at steady state. The model predicted the longer half-life observed for the split-thickness skin sample compared with that for the epidermis . Kubota and Maibach (10) developed a mathematical three-layer diffusion model and found the lag time and half-life after vehicle removal in epidermis and split-thickness skin were longer than those in the SC without viable layers .

However, the influence of interfaces in a laminated membrane on transfer kinetics has received little attention, due to the assumption that chemical equilibration via portioning is reached quickly enough in such a biphasic system that deeper investigation on to such issues as the interfacial barrier becomes unnecessary. For a rapid interfacial kinetics, a multi-phasic system understandably can be treated as a single-phasic problem (1). However, Albery et al. $(11,12)$ showed that significant freeenergy barriers exist during transport across a liquid-liquid interface. Once the interphase kinetic rate is slower, the impact of such interfacial barrier is noticeable on the diffusion profile. Hadgraft (13) provided a dimensionless parameter $\kappa$ to describe the rate of transport across an interface to characterize the interfacial kinetics. For an interphase between an organic substrate and an aqueous phase, the transport rate of the interphase

$$
\kappa=\frac{k_{\mathrm{I}} L}{D}
$$

Here $k_{\mathrm{I}}(\mathrm{m} / \mathrm{s})$ is the interfacial transfer rate of a substance from the organic substrate to the aqueous phase, $L$ is the thickness of the organic layer and $D$ is the diffusivity within the organic layer. For $\kappa \gg 1$, the interfacial transfer term is negligible and the system can be viewed as a single phase; while $\kappa=1$ indicates the interfacial effect dominating the transport characteristics and the interfacial barrier will exert a significant influence on drug release.

Cleek and Bunge (14) employed a finite twomembrane composite model dealing with a system comprising the SC and viable epidermis and an interphase between them. In addition, they found that the concentrations in the SC and viable epidermis at the interphase are related to the partition coefficients.

Actually, transdermal drug delivery is a transport process of drugs through a multi-laminar structure, e.g. from the patch to SC then to the viable epidermis, and finally penetrating into the blood. The process becomes complicated because of the flux barriers between interphases (patch/ SC, SC/viable epidermis). The interphases and their non-uniformity between any neighboring layers usually cause interruptions in transport flux and thus hinder the drug release effectiveness. In addition, an interphase by definition is the contact junction between two different phases, and its properties exhibit more or less some discrete or discontinuous nature, still a difficult problem in numerical modeling.

This paper develops a multilayer two dimensional finite-element model to account for effects of the interfacial kinetics on the drug delivery process. The study also develops a contact interface algorithm to deal with interface flux loss. The model assumes the patch is a non-limited reservoir. However, the algorithm can also be applied to the limited reservoir patch.

\section{Model and Algorithms}

The structure of the system consists of three parts (i) patch, (ii) SC and(iii) viable epidermis, and the 2D finite-element model is illustrated in Figs 1a and $b$. Here are the dimensions used in modeling (Table 1).

For computational consideration, the ratio of the patch length to the skin length of the section for analysis is chosen as 1:10 so that the edge effect can be neglected.

Based on the mass conservation law, we have

$$
\begin{aligned}
& M_{\text {stored }}+M_{\text {in-through-boundary }} \\
& \quad+M_{\text {out-through-boundary }}+M_{\text {generated }} \\
& \quad=0
\end{aligned}
$$



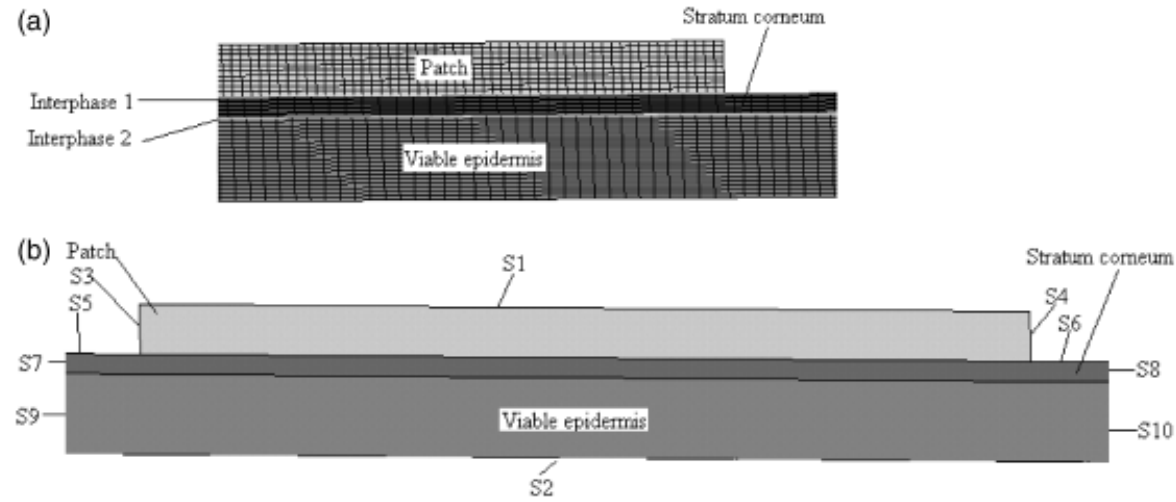

Fig. 1. The local finite element (a) and solid model (b) (just shown in part). The patch, stratum corneum and viable epidermis constitute the numerical three-layer model with 10 free surfaces (from S1 to S10) and two Interphases (Interphases 1 and 2).

TABLE 1. The dimensions used in modeling

\begin{tabular}{llll}
\hline Name & $\begin{array}{l}\text { Length } \\
(\mathrm{mm})\end{array}$ & $\begin{array}{l}\text { Thickness } \\
(\mu \mathrm{m})\end{array}$ & $\begin{array}{l}\text { Diffusion coefficient } \\
\left(\mathrm{mm}^{2} / \mathrm{s}\right)\end{array}$ \\
\hline Patch & 0.9 & 50 & $1 \times \mathrm{E}^{-9}$ (supposed) \\
SC & 9 & $20(15)$ & $\begin{array}{l}4.22 \times \mathrm{E}^{-8} \text { (supposed) } \\
\text { Viable epidermis }\end{array}$ \\
\hline
\end{tabular}

where $M_{\text {stored }}$ is material originally stored in the whole system, $M_{\text {in-through-boundary }}$ and $M_{\text {out- }}$ through-boundary are material moves in and out of the system, $M_{\text {generated }}$ is material generated by system during the process.

Applying the diffusion equilibrium equation to a unit solid, with no convection flow and no new substances generated due to chemical reactions during the process, we obtain

$$
\left[\frac{\partial C}{\partial t}\right]=\nabla \bullet(D \nabla C)
$$

Here, $C$ is the concentration, $D$ the diffusion coefficient and $t$ is time.

A $20 \mathrm{~h}$ diffusion process is then simulated with the following boundary conditions,

$$
\text { S1 }: \frac{\partial C}{\partial t}=0 \text { and } C(t)=C_{0}
$$

$\mathrm{S} 1, \mathrm{~S} 2, \mathrm{~S} 3, \mathrm{~S} 4, \mathrm{~S} 5, \mathrm{~S} 6, \mathrm{~S} 7, \mathrm{~S} 8, \mathrm{~S} 9, \mathrm{~S} 10: \frac{\partial c}{\partial t}=0$

$$
\text { and }\left.C(t)\right|_{t=0}=0
$$

and the interphase kinetics boundary conditions,

Interphase $1: J_{\text {Interphase } 1}$

$$
=\eta_{1}\left(C_{\text {Interphase 1+ }}-C_{\text {Interphase 1- }}\right)
$$

Interphase $2: J_{\text {Interphase } 2}$

$$
=\eta_{2}\left(C_{\text {Interphase 2+ }}-C_{\text {Interphase 2- }}\right)
$$

in which $J_{\text {Interphase } 1}$ and $J_{\text {Interphase } 2}$ are the mass flux at Interphases 1 and 2, the subscripts for $C$ are different interphasic concentrations. $\eta_{1}$ and $\eta_{2}$ are the mass transfer coefficients (TCs) (17).

We employed a contact algorithm to deal with interphase kinetics diffusion. For the interface drug diffusion scheme shown in Fig. 2, a concentration gradient across both contact and target layers is required. Thus the complete variation weak format is,

$$
\begin{aligned}
& \int_{\Omega}\left(\delta C\left[\frac{\partial C}{\partial t}\right]\right) d \Omega= \int_{\text {Interphase } 1} \delta C I_{\text {Interphase } 1} \mathrm{~d} s_{\text {Interphase } 1} \\
&+\int_{\text {Interphase } 2} \delta C J_{\text {Interphase } 2} \mathrm{~d} s_{\text {Interphase } 2} \\
&+\sum_{i=1}^{10} \delta C J_{i} \mathrm{~d} s_{i} \\
&\{J\}=-[D]\left\{\frac{\frac{\partial}{\partial x}}{\frac{\partial}{\partial y}}\right\}
\end{aligned}
$$

where, Diffusion coefficients matrix $[D]=\left[\begin{array}{ll}D_{x x} & 0 \\ 0 & D_{y y}\end{array}\right]$. Pseudo-homogenous porous media are assumed in the numerical model, i.e. $D_{x x}=D_{y y}$.

An Augmented Lagrange algorithm is applied to the interphases between different domains (with symbols \pm ). The contact variation weak formats is

$$
\delta \Psi=\int_{\Gamma}\left[\left(\lambda_{\mathrm{N}}+\varepsilon_{\mathrm{N}} g_{\mathrm{N}}\right) \delta g_{\mathrm{N}}+\left(\lambda_{\mathrm{T}}+\varepsilon_{\mathrm{T}} g_{\mathrm{T}}\right) \delta g_{\mathrm{T}}\right] \mathrm{d} A
$$


$\lambda_{\mathrm{T}} \delta g_{\mathrm{T}}$ is associated with tangential diffusion and $\varepsilon_{N} \delta g_{N}$ with normal diffusion.

All simulations are executed in software ANSYS 10.0.

\section{Results}

A transient delivery process with the patch maintaining a constant drug concentration is adopted in the present simulations. The time-evolving drug delivery is undergoing in current transient simulations during $20 \mathrm{~h}$. We first calculated and examined the effects of interphase barrier on multiplayer transdermal drug delivery using the interphacial Eqs. (6a) and (6b). For physical parameters in the numerical model, we assumed the diffusion coefficients as shown in Table 1. To investigate the influence of the interphasic barrier, the TC of Interphase 1 between patch and SC is assigned the values of $0,100,1$ and 0.001 $\mathrm{mm} / \mathrm{s}$, respectively. In addition, the constant concentration of the patch is $1 \mathrm{mg} / \mathrm{ml}$.

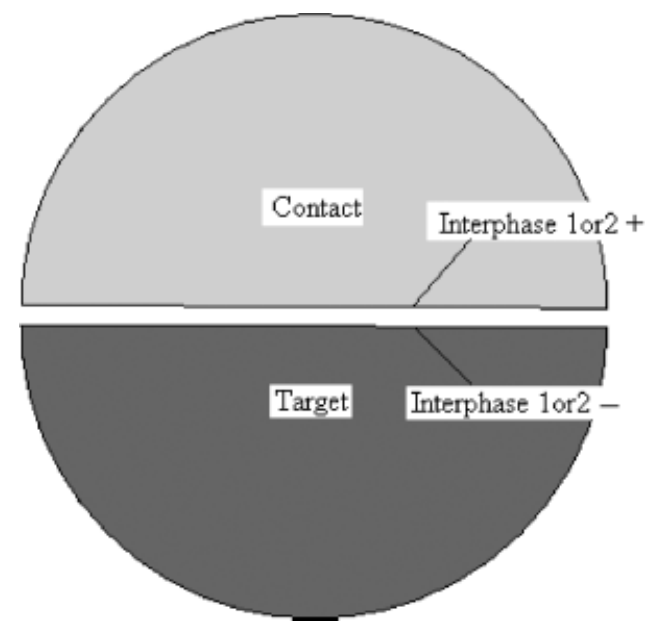

Fig.2. Illustration of the interphases between the Contact (+) and Target (-) layers. For Interphase 1, contact body is patch on Interphase $1+$ and target body is stratum corneum (SC) on Interphase 1 - and for Interphase 2, contact body is SC on Interphase 2+ and target body is viable epidermis on Interphase $2-$. A gap between the ' + ' and ' - 'sign interphases for illustration only.
Figure 3 shows the first case where the transfer coefficient for Interphase $1=0 \mathrm{~mm} / \mathrm{s}$ and Interphase $2=100 \mathrm{~mm} / \mathrm{s}$ so that a patch in the setup does not deliver drug to the skin at all, even after $20 \mathrm{~h}$ of physical diffusion time; there is a clear-cut drug concentration gradient with the maximum value at the patch layer and the minimum (0) level at the SC and viable epidermis layers. In other words, since the transfer coefficient is 0 , Interphase $1+$ could not diffuse the drug to Interphase $1-$.

The concentration and flux distribution after $20 \mathrm{~h}$ diffusion are shown in Fig. 4, where the transfer coefficients for both Interphases 1 and 2 are set at $100 \mathrm{~mm} / \mathrm{s}$ and the drug has been delivered through both Interphases 1 and 2 . The concentration gradually declines from the maximum to the minimum with an apparent isocontour distribution of concentration in Fig. 4a. The concentration distribution has high gradient around the corner of patch and SC, where the isocontour line is denser than other locations. The drug flux also shows the maxima at the corners in Fig. $4 b$.

The iso-contour distributions of drug concentration are an indication of a uniform distribution along both the length and thickness directions, except for the interacting corners between the patch and SC, thus allowing for the comparison of interphase barrier effects with interphase transfer coefficients. For more reliable prediction to avoid various edge effects, the middle section of the patch along the length direction is adopted along which seven characteristic points are chosen as shown in Fig. 5 to calculate and compare the concentration and flux distributions during drug diffusion. Locations 1, 2 and 3 are within the SC layer and Locations 4, 5, 6 and 7 are within the viable epidermis layer.

By setting the transfer coefficient of Interphase 1 at $100.0 \mathrm{~mm} / \mathrm{s}$, the drug diffusion concentration curves along the middle path with different interphase transfer coefficients for Interphase 2 are

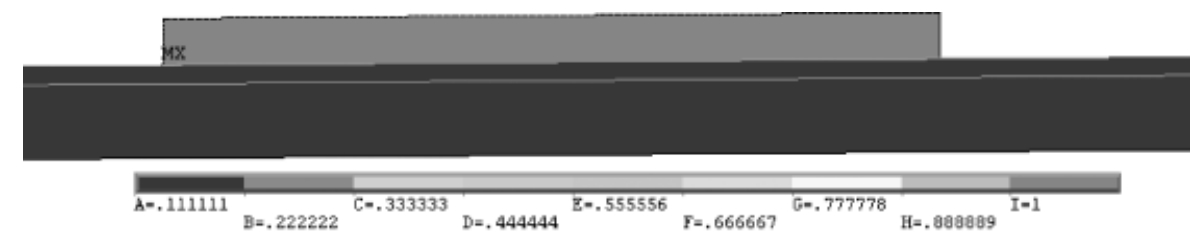

Fig. 3. Multilayer drug delivery results after $20 \mathrm{~h}$ diffusion shown in contour formats, when the interphase 1 transfer coefficient (TC) is supposed $0 \mathrm{~mm} / \mathrm{s}$ to show the contact transfer effect. The red suggests the maximum concentration value $1 \mathrm{mg} / \mathrm{mL}$ and blue the minimum $0 \mathrm{mg} / \mathrm{mL}$. No diffusion happens with the null interphase TC $(T C=0)$. 

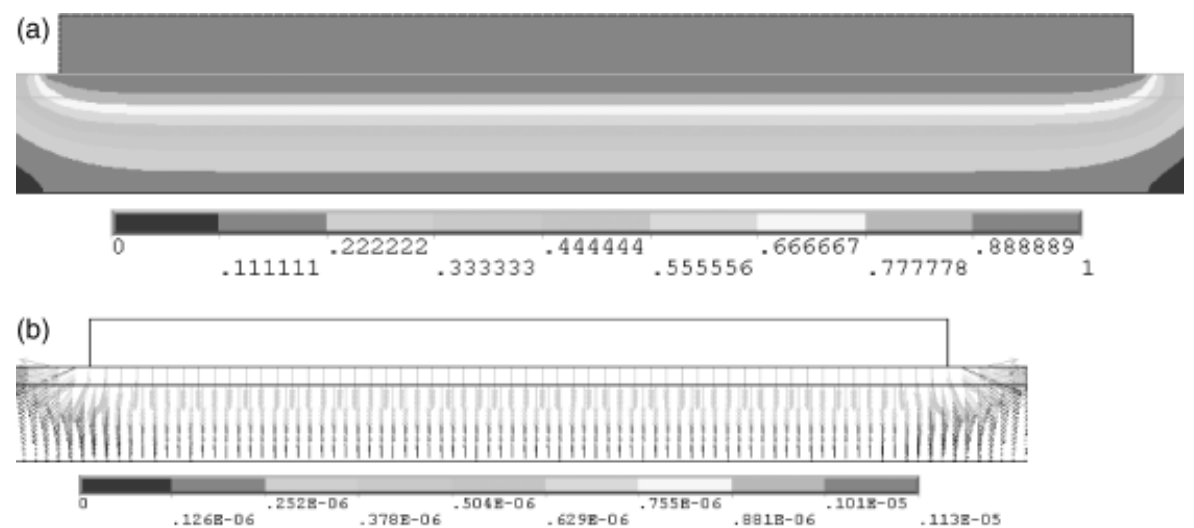

Fig. 4. Concentration (a) (unit: $\mathrm{mg} / \mathrm{ml}$ ) and flux (b) [unit: $\mathrm{mm} /\left(\mathrm{mm}^{2} \mathrm{~s}\right)$ ] contour by setting Interphase 2 TC $=100$ during $20 \mathrm{~h}$ physical diffusion. Drug has been delivered through Interphases 1 and 2. Concentration distribution has a high gradient around the corner of patch and SC, where the isocontour line is denser than other locations (a) and flux also show the maxima (red) at the corner (b).

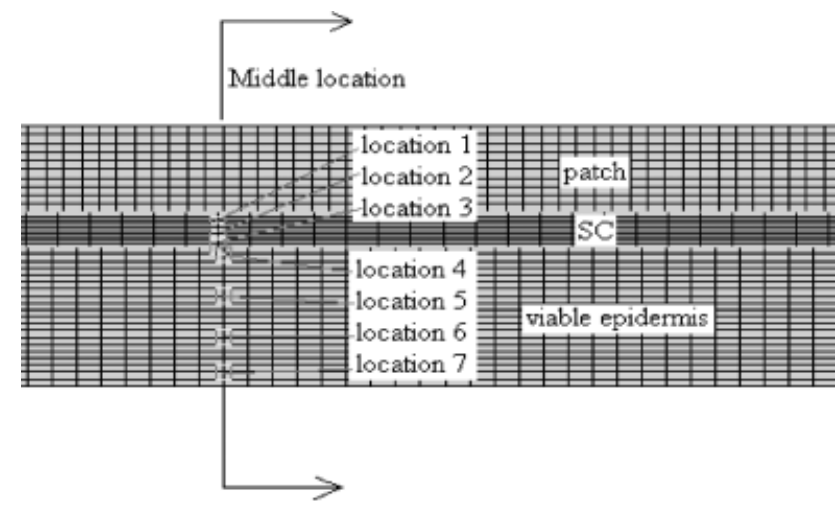

Fig. 5. The chosen seven locations (with empty square labeled) along the middle location line for comparison of the concentration and flux values [locations 1, 2, and 3 in the stratum corneum (SC) layer and location 4, 5, 6 and 7 in the viable epidermis]

shown in Fig. 6 and Fig. 7. The diffusion time is $20 \mathrm{~h}$ and the concentrations gradually move from Location 1 to 7 , where the transfer coefficient of Interphase 2 varies from $100.0 \mathrm{~mm} / \mathrm{s}$ in Fig. 6a, $1.0 \mathrm{~mm} / \mathrm{s}$ in Fig. $7 \mathrm{a}$ and $0.01 \mathrm{~mm} / \mathrm{s}$ in Fig. $7 \mathrm{~b}$.

\section{Discussion}

Transdermal delivery plays an important role in drug administration, where the functionalities of some drug molecules are apt to be offset because of being metabolized in conventional oral approach. In addition, dermal absorption also is a route by which environment chemical hazards can harm the human body. Mathematical models have been established to predict drug transport from the skin to inner organs or to investigate the drug delivery mechanism, examples including those by Guy and Hadgraft (18), Higuchi (19,
20), Bunge (21), Paul and McSpadden (22) et al. More details can be found from the excellent review papers on the subject by Kalia and Guy (1), and by Roberts and Anissimov (6), successively.

The present paper developed a new interphase contact algorithm using the finite-element method to account for the influences due to the interphase barrier in the whole system. A realistic model for transdermal delivery is established, including the structural layers of the patch, SC and viable epidermis into the finite element. Two interphases between the patch and SC, and between SC and viable epidermis, are identified on which the contact algorithm with the interphase transfer equation is employed.

To verify the interphase contact algorithm of diffusion, we first set the transfer coefficient into 0 of Interphase 1 between the patch and SC layer. The drug diffusion is hence inhibited and the concentrations show only maximum and minimum (0) with no transitional gradient in Fig. 3. This means the drug in the patch could not transfer to SC because of the null transfer coefficient. The as-expected concentration contours in Fig. 3 have substantiated the feasibility of the algorithm.

Then two non-zero constant transfer coefficients were assumed for both Interphases 1 and 2 in the model, and a $20 \mathrm{~h}$ transient diffusion process simulation was executed. As shown in Fig. 4a, the final concentration distributions provide the diffusion profiles at different locations and times around the mid-section of the system. The concentrations have a uniform diffusion front. However, the diffusions around the corner (right angle) between the patch and SC show 
Xing et al.

(a)
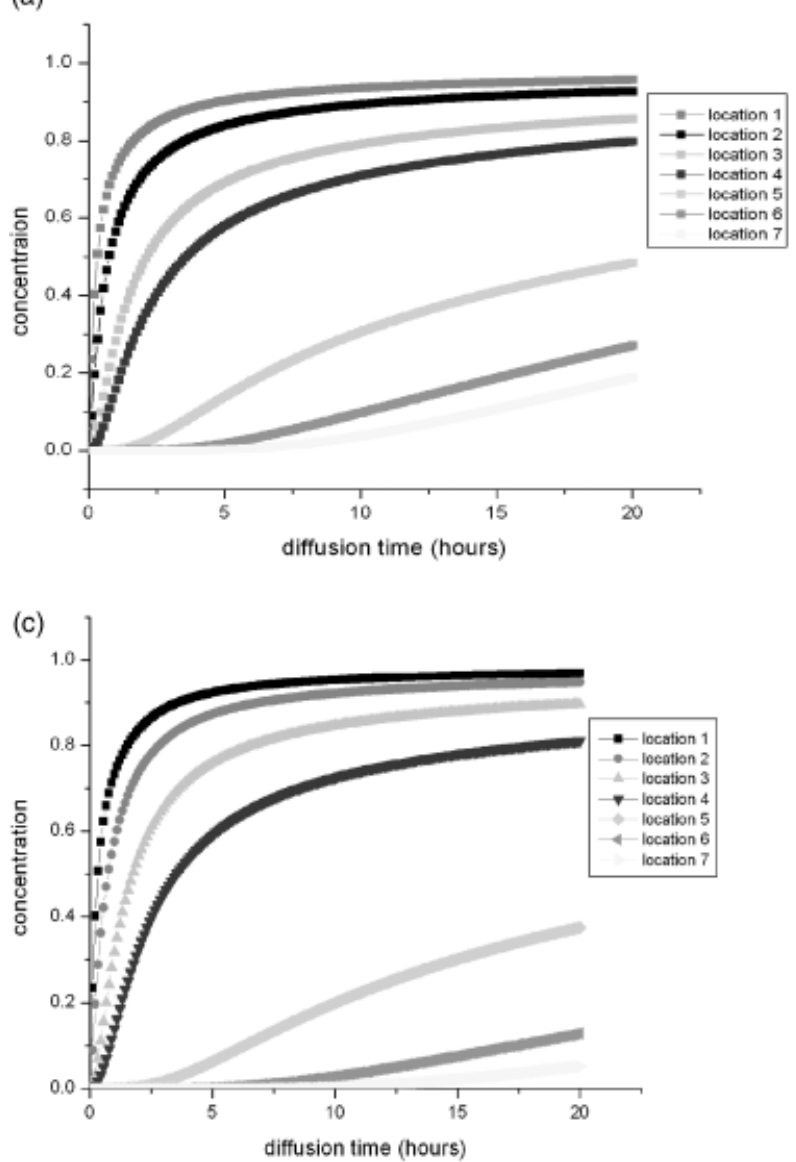

(b)

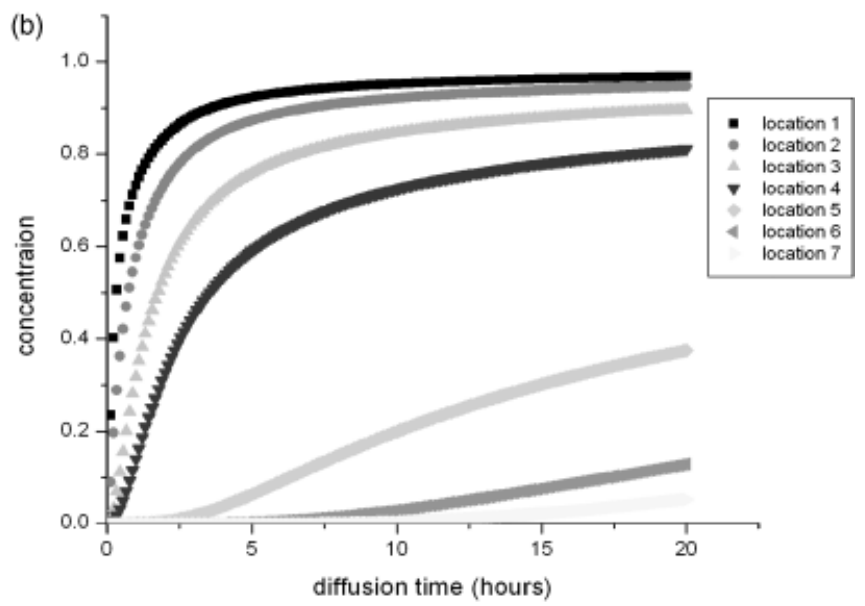

(d)

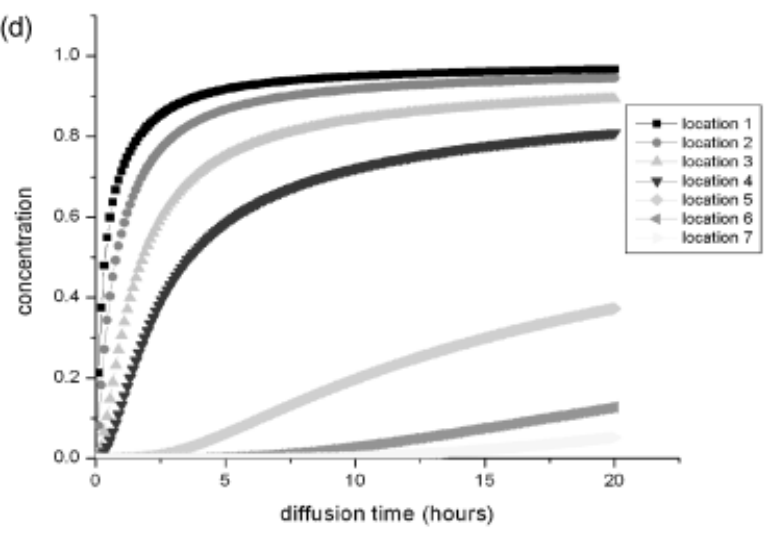

Fig. 6. Concentration changes at the seven locations by setting transfer coefficient (TC) $=100$ for Interphase 2, after 20h physical diffusion time. (a) $T C=100$ for Interphase 1; (b) TC $=1.0$ for Interphase 1; (c) TC $=0.01$ for Interphase $1 ;$ (d) TC $=0.0001$ for Interphase 1 . The X-axis presents time history (hour) and the Y-axis presents concentration.
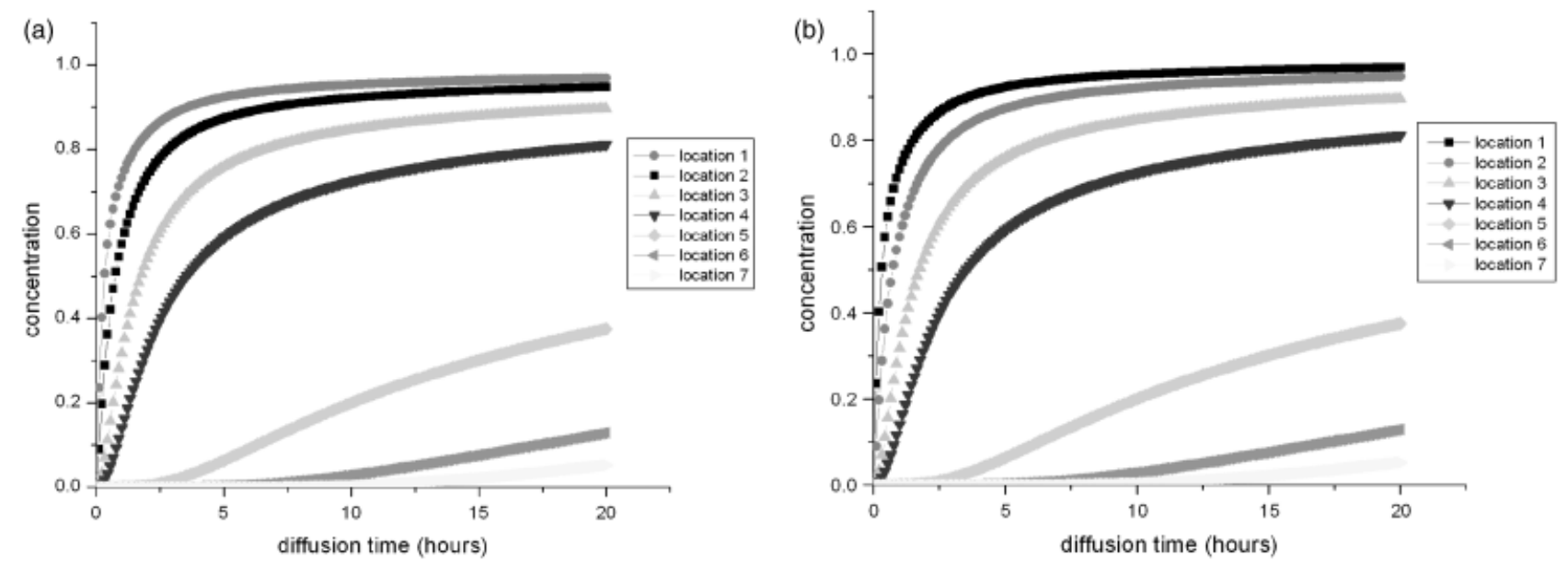

Fig.7. To investigate effects of transfer coefficient (TC) on delivery, concentrations are tracked at the seven locations by setting TC $=100$ for Interphase 1 after $20 \mathrm{~h}$ physical diffusion time. (a) TC $=1.0$ for Interphase 2; (b) TC $=0.01$ for Interphase 2. The X-axis presents time history (hour) and the $y$-axis presents concentration.

greater local concentration gradients and thus at the end higher local concentrations. The flux vector contour in Fig. $4 \mathrm{~b}$ also corresponds to concentration distributions. Maximum flux vector values are present around the corners, where the red vector represents the maximal value and the blue one the minimal.

To examine the effects of interphase barrier on drug delivery, adjustable interphase transfer coefficients are incorporated into the simulations. 

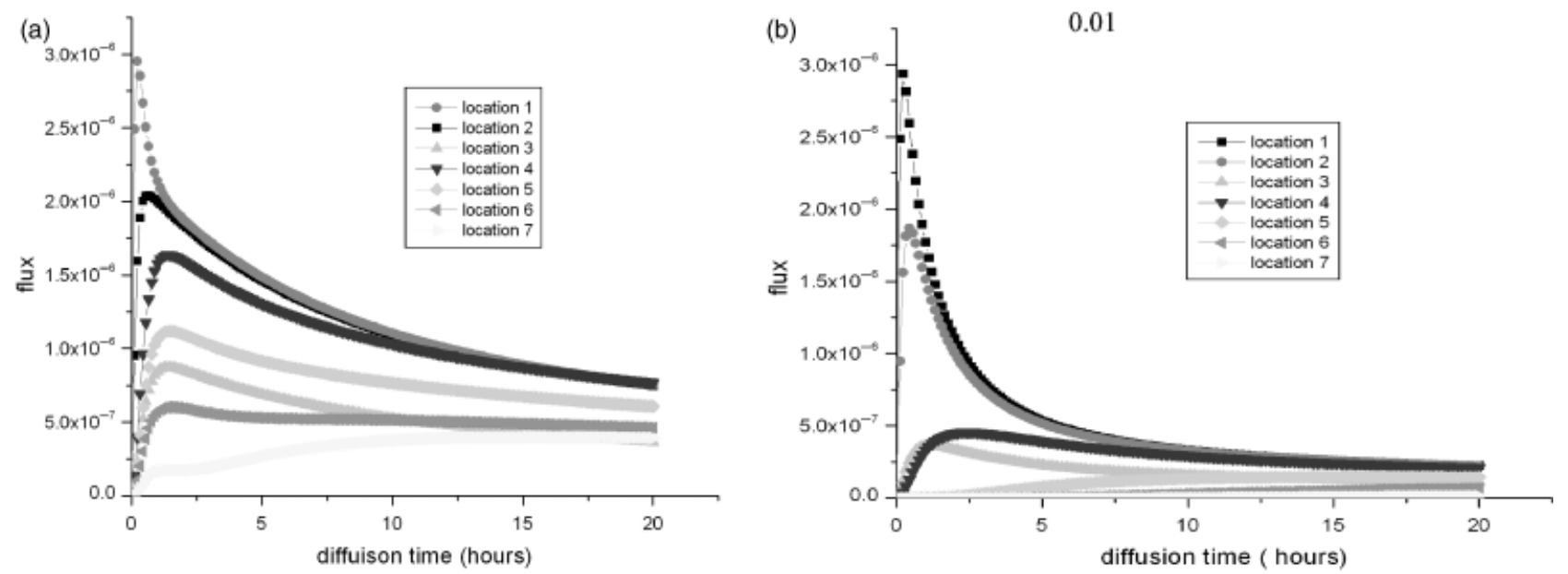

Fig. 8. As an important variable in drug delivery, the fluxes also compared at the seven locations along the middle line path with Interphase 1 transfer coefficients $100(\mathrm{a})$ and 0.01 (b) while Interphase 2 transfer coefficient (TC) =100 mm/s, after $20 \mathrm{~h}$ physical diffusion time. TC =100 for Interphase 1 ; $\mathrm{TC}=0.001$ for Interphase 1 .

Figure 3 provides such an initial validation, and Fig. 4 illustrates the distributions of both concentration and flux of the drug. A local path along the middle line of the model in Fig. 5 was adopted to compare the distribution and flux at seven different locations as functions of time (until $20 \mathrm{~h}$ ). Figure 6 presents such concentration distributions at different locations, where the transfer coefficient for Interphase 2 was fixed at $100 \mathrm{~mm} / \mathrm{s}$ while the value for Interphase 1 changes from $100,1,0.1$, to $0.01 \mathrm{~mm} / \mathrm{s}$. The curves for the SC layer at Locations 1, 2 and 3 first show a quick rise in the first $5 \mathrm{~h}$, then stabilized and approaching their corresponding asymptotes. However, the concentrations in the viable epidermis layer exhibit an initial stagnation resulting from the long diffuse time from the patch.

More specific information can be obtained from Fig. 6. By altering the transfer coefficients of Interphase 1 between the patch and SC, one can control or fine-tune the diffusion process and the concentration or flux distributions at a given time, which is clearly advantageous for optimal drug effectiveness.

Similar results are shown in Fig. 7 with fixed transfer coefficient $100 \mathrm{~mm} / \mathrm{s}$ at Interphase 2 and varying values for Interphase 1. Figure 8 also illustrates the influence of the transfer coefficients on the diffusion process, except that the transfer coefficient at Interphase 1 is fixed at $100 \mathrm{~mm} / \mathrm{s}$ and that for Interphase 1 is allowed to change. The drug flux is much smaller in transfer coefficient 0.01 (Fig. 7b) than in 100 (Fig. 7a). Also from Fig. 6, Fig. 7, Fig. 8, the larger transfer coefficients provided more robust control in the diffusion process.
The simulation results and the interphase contact algorithm in this study demonstrate the possibility of a controllable transdermal drug delivery approach. Furthermore, the algorithm can deal with complicated geometrical conditions, which is difficult for classical analytical solution. We will incorporate these into our future work.

$$
\begin{gathered}
\text { Interphase } 1: J_{\text {Interphase } 1}=\eta_{1}\left(\kappa_{1} C_{\text {Interphase } 1+}\right. \\
\left.-C_{\text {Interphase } 1-}\right) \\
\text { Interphase } 2: I_{\text {Interphase } 2}=\eta_{2}\left(\kappa_{2} C_{\text {Interphase 2+ }}\right. \\
\left.-C_{\text {Interphase 2- }}\right)
\end{gathered}
$$

Here $\kappa_{1}$ and $\kappa_{2}$ are parameters related to partition coefficients.

This technique, once further improved by incorporating the partitions coefficients (8a) and (8b) and the other remaining interphase barrier factors into the model and calibrated with experiment data, may provide a robust tool for drug and patch design in transdermal drug delivery applications. Taken together, however, modeling provides but one step, a step that requires experimental validation.

\section{References}

1. Kalia YN, Guy RH. Modeling transdermal drug release. Adv Drug Deliv Rev 2001; 48: 159-172.

2. Rim JE, Pinsky PM, van Osdol WW. Finite element modeling of coupled diffusion with partitioning in 


\section{Xing et al.}

transdermal drug delivery. Ann Biomed Eng 2005; 33: 1422-1438.

3. Hui X, Wester RC, Zhai H, Maibach HI. Chemical partitioning into powered human stratum corneum: a useful in vitro model for studying interaction of chemicals and human skin. In: Bronaugh RL, Maibach HI, eds. Percutaneous absorption. Boca Raton: Taylor \& Francis Group, 2005: 291-302.

4. Scheuplein RJ. Percutaneous absorption: theroetical aspects. In: Mauvais-Jarvis P, Vickers CF, Wepierre J, eds. London: Academic Press, 1980: 1-17.

5. Truskey GA, Yuan F, Katz DF. Transport phenomena in biological system. Upper Saddle River: Pearson Prentice Hall, 2004: P263-P264.

6. Roberts MS, Anissimov YG. Mathematical models in percutaneous absorption. In: Bronaugh Robert L., Maibach Howard I., eds. Percutaneous absorption. Boca Raton: Taylor \& Francis Group, 2005: 1-44.

7. Hostynek JJ, Reifenrath WG, Melendres JL, Magee PS. Exogenous dermatology. In: Surber C, Elsner P, Bircher AJ, eds. Current problems in dermatology. S. Karger Verlag, 1995: 139-145.

8. Kubota K, Maibach HI. A compartment model for percutaneous absorption: compatibility of lag time and steady-state flux with diffusion model. J Pharm Sci 1992; 81: 863-865.

9. Kubota K, Sznitowska M, Maibach HI. Percutaneous absorption: a single-layer model. J Pharm Sci 1993; 82: 450-456.

10. Kubota K, Maibach HI. Significance of viable skin layers in percutaneous permeation and its implication in mathematical models: theoretical consideration based on parameters for betamethasone 17-valerate. J Pharm Sci 1994; 83: 1300-1306.

11. Albery WJ, Couper AM, Hadgraft J, Ryan C. Transport and kinetics in two phase systems. J Chem Soc, Faraday Trans 1974; 1: 1124-1131.

12. Albery WJ, Burke JF, Leffler EB, Hadgraft J. Interfacial transfer studied with a rotating diffusion cell. J Chem Soc, Faraday Trans 1976; 1: 1618-1626.

13. Hadgraft J. Calculations of drug release rates from controlled release devices. The slab. Int J Pharm 1979; 2: 177-194.
14. Cleek RL, Bunge AL. A new method for estimating dermal absroption from chemical exposure. 1. General approach. Pharm Res 1993; 10: 497-506.

15. Holbrook KA, Odland GF. Regional differences in the thickness (cell layers) of the human stratum corneum: an ultrastructural analysis. J Invest Dermatol 1974; 62: 415-422.

16. Timar F, Soos G, Szende B, Horvath A. Interdigitation index - a parameter for differentiating between young and older skin specimens. Skin Res Technol 2000; 6: 17-20.

17. Bird RB, Steeart WE, Lightfoot EN. Notes on transport phenomena. New York: John Wiley \& Sons Inc, 1958: P313-324.

18. Guy RH, Hadgraft J. Rate control in transdermal delivery? Int J Pharmacol 1992; 82: R1-R6.

19. Higuchi T. Physical chemical analysis of percutaneous absorption process from creams and ointments. J Soc Cosmet Chem 1960; 11: P85-P97.

20. Higuchi T. Rate of release of medicaments from ointment bases containing drugs in suspension. J Pharm Sci 1961; 50: 874-875.

21. Bunge AL. Release rates from topical formulations containing drugs in suspension. J Control Release 1998; 52: 141-148.

22. Paul DR, McSpadden SK. Diffusional release of a solute from a polymer matrix. J Membr Sci 1976; 1: 33-48.

Address:

Prof Howard I. Maibach

Department of Dermatology

School of Medicine

University of California San Francisco

Box 0989, Surge 110

San Francisco, CA 94143-0989

USA

Tel: +14154762468

Fax: +1 4157535304

e-mail: MaibachH@derm.ucsf.edu 